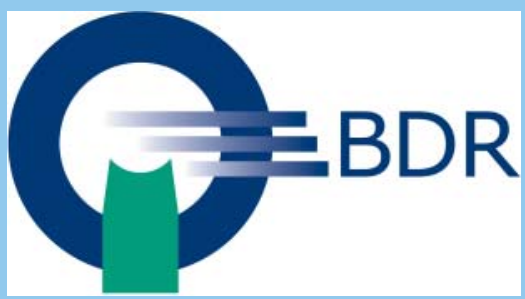

\title{
Strukturwandel in der Radiologie - Antworten des BDR
}

Unter diesem Motto trafen sich in diesem Jahr zum zweiten Mal die BDR-Landesvorsitzenden mit dem Vorstand als Länderausschuss in Berlin.

Dr. Reichmuth, der Past-LänderausschussVorsitzende, begrüßt in Vertretung seines Nachfolgers Dr. Michael Herzau, Thüringen, die Teilnehmerlnnen.

Neben den Dauerbrennerthemen (Weiterentwicklung EBM/GOÄneu/WBO) war es vor allem der Vortrag des Steuerberaters Klaus Weippert, dessen Morgen-Referat zur Verfahrensdokumentation eine Art Weckruf war.

\section{Verfahrensdokumentation - die betriebs- wirtschaftlichen Stolpersteine}

Herr Weippert weist eindringlich auf die Dokumentationspflicht und die notwendige Verfahrensdokumentation hin. Schwerpunkt ist die seit 2015 geltende GoPD und die Konsequenzen für die digitale Buchführung. Durch erste Urteile des BFH wird die Kompetenz der Betriebsprüfer gestärkt, die dieses auch anwenden. Dies betrifft insbesondere die digitalen Abläufe des wirtschaftlichen Teils einer jeden Arztpraxis.

Das Thema wurde intensiv diskutiert. In der August-Ausgabe des RADIOLOGEN werden wir hierzu eine ausführliche Dokumentation und Handlungsanweisung veröffentlichen.

Die Berichte aus den Ländern nehmen i. d. R. den Großteil der Diskussion ein. Hierbei kommt es immer zum intensiven Austausch und der Erfahrung, dass die Umsetzung der Honorarordnung trotz Kollektivvertrags aufgrund der föderalen Strukturen gänzlich verschieden sein kann.
Diagnostik-Fächer kandidieren auf eigenen DVÄD-Listen

Baden-Württemberg macht es vor: Dr. Köpke hat maßgeblichen Anteil an der erfolgreichen Initiative für eine eigene Kandidatenliste in Baden-Württemberg. Aus den Fachgruppen Pathologie, Mikrobiologie, Labormedizin und Radiologie hat sich eine KandidatInnen-Liste geformt, die im Herbst zu den Kammerwahlen antreten. Im Saarland existiert eine solche Listenverbindung schon seit Langem. Die Interessen der DiagnostikFächer können nur Diagnostiker vertreten!

\section{Kontrastmittel - Therapiefreiheit wahren!}

Der BDR vertritt weiterhin die bereits mehrfach veröffentlichte und in der diesjährigen Delegiertenversammlung auch bestätigte Position. Die diagnostische und therapeutische Freiheit des Radiologen in der Auswahl der verwendeten Kontrastmittel muss gewährleistet sein. Bürokratie und Sparzwänge sollen das sensible Verhältnis zwischen Patient und Arzt nicht belasten. Wie dies in unterschiedlichen Modellen, die es mittlerweile in den verschiedenen LänderKVs gibt, umgesetzt wird, bleibt den Landesvorsitzenden vorbehalten. Die Dumpingpreise für Kontrastmittel in den Kliniken entziehen dem ambulanten Sektor Geld und sind so nicht hinzunehmen.

Festgelegt wurde, dass aufgrund der wirkstoffübergreifenden Ausschreibung in Berlin eine Feststellungsklage gegenüber den Prüfstellen erwogen wird.

\section{Tue Gutes - Radiologen unterstützen Pathologen}

Bereits mehrfach haben wir über das Telekonsultationsprojekt „Telebridging“ des Auricher Pathologen Dr. Stauch berichtet.
Er wirbt für die ehrenamtliche Mitarbeit von RadiologInnen im Telekonsultationsdienst für Entwicklungsländer „I PATH“, siehe hierzu DER RADIOLOGE 2017, S.990.

\section{BDR und DRG - eine gute Verbindung}

Die Zusammenarbeit zwischen wissenschaftlicher Gesellschaft und Berufsverband verbessert sich kontinuierlich. Im Hintergrund findet dies auf der Arbeitsebene in den jeweiligen Gremien statt, präsent und für alle sichtbar aber im Rahmen des Röntgenkongresses. Auch 2018 waren die vom BDR mitverantworteten Veranstaltungen „Radiologie in Klinik und Praxis“ (siehe auch die Juni-/Juli-Ausgaben des RADIOLOGEN) bestens besucht, widmeten sie sich doch gemeinsamen Themen, die sowohl im ambulanten wie im stationären Bereich virulent sind.

\section{Radiologie und Industrie}

Anhand des Themas „Dosismanagement“ wird deutlich, dass in der Zusammenarbeit mit Herstellerfirmen auch Vorsicht geboten ist. Der BDR warnt die Mitglieder vor voreiligen kostenintensiven Aktivitäten. Der BDR hat hierzu eine Empfehlung formuliert, siehe DER RADIOLOGE, 2018, S. 691.

Dr. Reichmuth beendete die Sitzung wie immer pünktlich und verabschiedete sich aus seiner Funktion als LänderausschussVorsitzender - dem Gremium bleibt er als Landesvorsitzender Berlins natürlich erhalten. Die Landesvorsitzenden dankten ihm mit großem Applaus.

Der nächste Länderausschuss findet im November 2018 statt.

Sönke Schmidt/Sabine Lingelbach 